\title{
Piecewise continuous distribution function method: Fluid equations and wave disturbances at stratified gas
}

\author{
Vereshchagin D.A., S. B. Leble \\ Theoretical Physics Department, Kaliningrad State University, \\ 236041, Kaliningrad, Al. Nevsky str. 14. \\ Theoretical Physics and Mathematical Methods Department, \\ Technical University of Gdansk, ul, Narutowicza 11/12, Gdansk, Poland, \\ leble@mifgate.pg.gda.pl
}

15th November 2018

\begin{abstract}
Wave disturbances of a stratified gas are studied. The description is built on a basis of the Bhatnagar - Gross - Krook (BGK) kinetic equation which is reduced down the level of fluid mechanics. The double momenta set is introduced inside a scheme of iterations of the equations operators, dividing the velocity space along and opposite gravity field direction. At both half-spaces the local equilibrium is supposed. As the result, the momenta system is derived. It reproduce Navier-Stokes and Barnett equations at the first and second order in high collision frequencies. The homogeneous background limit gives the known results obtained by direct kinetics applications by Loyalka and Cheng as the recent higher momentum fluid mechanics results of Chen, Rao and Spiegel. The ground state declines from exponential at the Knudsen regime. The WKB solutions for ultrasound in exponentially stratified medium are constructed in explicit form, evaluated and plotted.
\end{abstract}




\section{Introduction.}

\section{Devoted to D.A. Vereshchagin memory}

There are gas dynamics problems at which it is necessary to use a basis going out of traditional Navier - Stokes hydrodynamics. It is connected to a break of the condition: $K n=l / L<<1$, where $K n$ - Knudsen number, $l$ - free particle path, and $L$ - characteristic scale of non-homogeneity of a problem. Perhaps, the first work, in which a wave disturbance in a gas was investigated from the point of view of more general kinetic approach was the work of Wang Chang and Uhlenbeck [1]. The authors have offered a method of a dispersion relation construction in a homogeneous gas directly from Boltzmann equation.

The further theoretical and experimental researches [3] - 15] on sound propagation in a homogeneous gas have shown, that at Knudsen numbers of the unit order the waves behavior considerably differs from ones predicted on a basis of Navier - Stokes equations. These researches revealed two essential features: first, the perturbations keep wave properties at more large values of $K n$, than it could be assumed on a basis of the classical hydrodynamic description. Secondly, at $K n \geq 1$ such concepts as a wave vector and frequency of a wave become ill-determined. May be the most adequate results that reproduce experiments [3] almost in all the range were obtained in [12. It is more difficult to explore the case, when the Knudsen number is non-uniform in space or in time and passes the Knudsen regime area. The statement and the solution of such problems should definitely be based on a kinetic equations or their advance model analogues.

Quite recently interest to the problems has grown again in connection with general fluid mechanics development [16, 17, 18, 19, 20]. It was pushed by more deep understanding of perturbation theory (so-called nonsingular perturbations), see, e.g. [21].

In his paper we consider the gas medium, stratified exponentially in gravity field, directed along $\mathrm{z}$ axis. In means that the Knudsen number also depends on z: $K n(z)$. We continue to develop the method [18 that goes up to the pioneering paper of Lees 22]. The construction of analytical solutions of the model kinetic equation Bhatnagar - Gross - Krook (BGK) is extracted via separate representation of the distribution function as the local equilibrium one but with different momenta sets at positive and negative velocity component $v_{z}$ subspaces. 
Thus, the set of parameters determining a state of the gas increases twice. Such number of parameters of the distribution function (6) results in that the distribution deviates from a local-equilibrium and accordingly widen hydrodynamics. In the range of small Knudsen numbers $l<<L$ we have $\hat{M}_{n}^{+}=\hat{M}_{n}^{-}$and distribution function (6) passes to local equilibrium one, giving a solution of the Navier-Stokes hydrodynamical regime. For big Knudsen numbers the formula (6) gives a solution of so-called collisionless problems. Similar ideas have resulted successfully in a series of problems. For example, in papers [22] - 27] a method of discontinuous distribution functions was used for the description of a flat and cylindrical (neutral and plasma) flows [22 - 27]. For a flat problem the surface of break in space of speeds was determined by the same natural condition $V_{z}=0$, and in a cylindrical case $V_{r}=0$, where $V_{z}$ and $V_{r}$, vertical and radial component of speed of particles respectively. The problem of a disturbance launched by a pulse movement of plane [27] was solved similarly. In a problem of a shock wave structures [27, 28, 29] the solution was represented as a combination of two locally equilibrium functions, one of which determines the function before front of a wave, and another - the tail. In a problem of condensation and evaporation of drops of any size [30, 31] a break surface was determined by so-called "cone of influence", thus all particles were divided to two types: flying "from a drop" and flying "not from a drop".

At the first two sections we derive the basic equations using the iterations in the evolution operator along the idea of the nonsingular perturbation method. Next (Sec. 4) we analyze the transition to a limiting case of a gas disturbances at large collision frequencies up to the Barnett case. Next (Sec. 5 ) we check the free molecular flow limit, demonstrating the declinations from the exponential behavior of the "atmosphere" gas density [32, ?]. At the final section we construct solutions of the main momenta system by the method VKB, considering the wave scale less than the scale of the inhomogeneity.

\section{Linearized BGK equation}

The kinetic equation with the model collision integral in BGK form looks like:

$$
\frac{\partial f}{\partial t}+\vec{v} \frac{\partial f}{\partial \vec{r}}-g \frac{\partial f}{\partial v_{z}}=\nu\left(f_{l}-f\right)
$$


here $f$-distribution function of a gas, $t$-time, $\vec{v}$ - velocity of a particle of the gas, $\vec{r}$ - its coordinate vector,

$$
f_{l}=\frac{n}{\pi^{3 / 2} v_{T}^{3}} \exp \left(-\frac{(\vec{v}-\vec{U})^{2}}{v_{T}^{2}}\right)
$$

- local-equilibrium distribution function, $H$ - a scale of inhomogeneity (in atmospheric models $H=k T / m g), v_{T}=\sqrt{2 k T / m}$ - average thermal velocity of movement of particles of gas, $\nu=\nu_{0} \exp (-z / H)$ - effective frequency of collisions between particles of gas at height $z$. It is supposed, that density of gas is denoted as $n$, its average speed $\vec{U}=\left(u_{x}, u_{y}, u_{z}\right)$ and temperature $T$ are functions of time and coordinates.

Considering small gas perturbations, we shall approximate a distribution function $f$ as:

$$
f(t, \vec{r}, \vec{v})=f_{0}(z, \vec{v})(1+\varphi(t, \vec{r}, \vec{v})), \quad|\varphi|<<1 .
$$

Here $f_{0}$ - equilibrium Maxwell - Boltzmann distribution function , and $\varphi-$ dimensionless small-amplitude perturbation to equilibrium distribution function. For thermodynamic parameters of a gas we shall write:

$$
n=n_{0}\left(1+n^{\prime}\right), \quad \vec{U}=\vec{U}^{\prime} v_{T}, \quad T=T_{0}\left(1+T^{\prime}\right),
$$

where $n^{\prime}, \overrightarrow{U^{\prime}}$ and $T^{\prime}$ - dimensionless small additives to equilibrium density, average speed and temperature gas respectively. Expanding the local equilibrium function $f_{l}$ up to the first order by small amplitudes and taking into account, that equilibrium function $f_{0}$ identically satisfies the kinetic equation (11) we obtain linearized equation BGK.

We shall consider one-dimensional case of the equation:

$$
\frac{\partial \varphi}{\partial t}+v_{z} \frac{\partial \varphi}{\partial z}-g \frac{\partial \varphi}{\partial v_{z}}=\nu\left\{\sum_{n=1}^{3} M_{n}(t, z) \chi_{n}(\vec{v})-\varphi\right\} \text {. }
$$

Here $\chi_{n}(\vec{v})$ - eigen functions of the linearized collisions operator:

$$
\begin{array}{rlrl}
\chi_{1} & =1, & \chi_{4} & =\frac{2}{\sqrt{5}} \frac{v_{z}}{v_{T}}\left(\frac{v^{2}}{v_{T}^{2}}-\frac{5}{2}\right), \\
\chi_{2} & =\sqrt{2} \frac{v_{z}}{v_{T}}, & \chi_{5} & =\frac{1}{\sqrt{3} v_{T}^{2}}\left(v^{2}-3 v_{z}^{2}\right), \\
\chi_{3} & =\sqrt{\frac{2}{3}}\left(\frac{v^{2}}{v_{T}^{2}}-\frac{3}{2}\right), \chi_{6}=\sqrt{\frac{6}{5}} \frac{v_{z}}{v_{T}^{3}}\left(v^{2}-\frac{5}{3} v_{z}^{2}\right) .
\end{array}
$$


$M_{n}$ - the moments of distribution function determined through scalar products:

$$
M_{n}(t, z)=<\chi_{n}, \varphi>\equiv \frac{1}{\pi^{1 / 2} v_{T}^{3}} \int d \vec{v} \exp \left(-v^{2} / v_{T}^{2}\right) \cdot \chi_{n}(\vec{v}) \varphi(t, z, \vec{v}) .
$$

In linear approach the moments $M_{n}$ are linked to the thermodynamic variables as:

$$
M_{1}=\frac{n-n_{0}}{n_{0}}, \quad M_{2}=\sqrt{2} \frac{u_{z}}{v_{T}}, \quad M_{3}=\sqrt{\frac{2}{3}} \frac{T-T_{0}}{T_{0}} .
$$

\section{A method of piecewise continuous distri- bution function in linear approximation.}

Following the idea of a method of piecewise continuous distribution functions 32 let's search the solution $\varphi$ of the equations (2) as a combination of two locally equilibrium distribution functions, each of which gives the contribution to the corresponding area of the velocity subspace as follows:

$$
\varphi= \begin{cases}\varphi^{+}=\hat{M}_{1}^{+}+\sqrt{2} \frac{v_{z}}{v_{T}} \cdot \hat{M}_{2}^{+}+\sqrt{\frac{2}{3}}\left(\frac{v^{2}}{v_{T}^{2}}-\frac{3}{2}\right) \hat{M}_{3}^{+}, & v_{z} \geq 0 \\ \varphi^{-}=\hat{M}_{1}^{-}+\sqrt{2} \frac{v_{z}}{v_{T}} \cdot \hat{M}_{2}^{-}+\sqrt{\frac{2}{3}}\left(\frac{v^{2}}{v_{T}^{2}}-\frac{3}{2}\right) \hat{M}_{3}^{-}, & v_{z}<0\end{cases}
$$

The parameters $\hat{M}_{n}^{ \pm}$of locally equilibrium distributions functions are linked to the correspondent density, average speed and temperature and three higher moments. This idea of the method of two-fold distribution functions of (6) further is realized as follows. Multiplying the BGK equation (2) by the

eigen functions (3) with the account of (4), calculating integrals we obtain a 
system of the differential equations for the moments $M_{n}$ :

$$
\begin{aligned}
& \frac{\partial M_{1}}{\partial t}+\frac{v_{T}}{2} \frac{\partial M_{2}}{\partial z}-\frac{v_{T}}{2 H} M_{2}=0, \\
& \frac{\partial M_{2}}{\partial t}+v_{T} \frac{\partial}{\partial z}\left(M_{1}+M_{3}-\frac{2}{3} M_{5}\right)-\frac{v_{T}}{H}\left(M_{3}-\frac{2}{3} M_{5}\right)=0, \\
& \frac{\partial M_{3}}{\partial t}+\frac{v_{T}}{3} \frac{\partial}{\partial z}\left(M_{2}+M_{4}\right)-\frac{v_{T}}{3 H} M_{4}=0, \\
& \frac{\partial M_{4}}{\partial t}+v_{T} \frac{\partial}{\partial z}\left(\frac{5}{2} M_{3}-\frac{1}{6} M_{5}\right)-\frac{v_{T}}{2 H} M_{5}=-\nu(z) M_{4}, \\
& \frac{\partial M_{5}}{\partial t}+v_{T} \frac{\partial}{\partial z}\left(-M_{2}-\frac{2}{5} M_{5}+\frac{9}{5} M_{6}\right)+\frac{v_{T}}{H}\left(\frac{2}{5} M_{4}-\frac{9}{5} M_{6}\right)=-\nu(z) M_{5}, \\
& \frac{\partial M_{6}}{\partial t}+\frac{v_{T}}{3} \frac{\partial M_{5}}{\partial z}+\frac{v_{T}}{6 H} M_{5}=-\nu(z) M_{6} .
\end{aligned}
$$

The moments $M_{n}$ of the distribution function are connected to parameters $\hat{M}_{n}^{ \pm}$of the two-fold distribution functions (6) by the relations:

$$
\begin{aligned}
M_{1} & =M_{1}^{+}+M_{2}^{-}, & M_{4} & =-M_{1}^{-}+\frac{7}{2} M_{3}^{-}, \\
M_{2} & =\sqrt{2}\left(M_{1}^{-}+\frac{1}{2} M_{2}^{+}+\frac{1}{2} M_{3}^{-}\right), & M_{5} & =-\frac{1}{\sqrt{3}} M_{2}^{-}, \\
M_{3} & =\sqrt{\frac{3}{2}}\left(\frac{1}{3} M_{2}^{-}+M_{3}^{+}\right), & M_{6} & =\sqrt{\frac{6}{5}}\left(\frac{1}{3} M_{1}^{-}+\frac{1}{2} M_{3}^{-}\right) .
\end{aligned}
$$

Here the following notations are introduced:

$$
\begin{array}{ll}
M_{1}^{+}=\frac{1}{2}\left(\hat{M}_{1}^{+}+\hat{M}_{1}^{-}\right), & M_{1}^{-}=\frac{1}{2 \sqrt{\pi}}\left(\hat{M}_{1}^{+}-\hat{M}_{1}^{-}\right), \\
M_{2}^{+}=\frac{1}{\sqrt{2}}\left(\hat{M}_{2}^{+}+\hat{M}_{2}^{-}\right), & M_{2}^{-}=\frac{1}{\sqrt{2 \pi}}\left(\hat{M}_{2}^{+}-\hat{M}_{2}^{-}\right), \\
M_{3}^{+}=\frac{1}{\sqrt{6}}\left(\hat{M}_{3}^{+}+\hat{M}_{3}^{-}\right), & M_{3}^{-}=\frac{1}{\sqrt{6 \pi}}\left(\hat{M}_{3}^{+}-\hat{M}_{3}^{-}\right) .
\end{array}
$$


The feedback between parameters $M_{n}^{ \pm}$and the moments $M_{n}$ is determined from (8) and looks like:

$$
\begin{array}{lll}
M_{1}^{+}=M_{1}+\sqrt{3} M_{5}, & M_{1}^{-}=\frac{3}{10} \sqrt{5}\left(-M_{4}+\frac{7}{6} \sqrt{6} M_{6}\right), \\
M_{2}^{+}=\sqrt{2} M_{2}+\frac{2}{5} \sqrt{5} M_{4}+\frac{12}{5} \sqrt{\frac{10}{3}} M_{6}, & M_{2}^{-}=-\sqrt{3} M_{5}, \\
M_{3}^{+}=\left(\sqrt{\frac{2}{3}} M_{4}+\frac{\sqrt{3}}{3} M_{5}\right), & M_{3}^{-}=\frac{1}{5} \sqrt{5}\left(M_{4}+\frac{1}{2} \sqrt{6} M_{6}\right) .
\end{array}
$$

\section{A limiting case of large collision frequen- cies.}

Within limits of small Knudsen numbers, from the equations (77) it follows, that $M_{4}, M_{5}, M_{6}<<M_{1}, M_{2}, M_{3}$. Then in a limit $\nu \rightarrow \infty(K n \ll 1$, hydrodynamical limit) we have $M_{4}, M_{5}, M_{6} \rightarrow 0$ and the system (17) tends to the linearized Euler's system:

$$
\begin{aligned}
& M_{1 t}+\frac{v_{T}}{2} M_{2 z}-\frac{v_{T}}{2 H} M_{2}=0, \\
& M_{2 t}+v_{T}\left(M_{1}+M_{3}\right)_{z}-\frac{v_{T}}{H} M_{3}=0, \\
& M_{3 t}+\frac{v_{T}}{3} M_{2 z}=0,
\end{aligned}
$$

where the bottom indices $t, z$ denote partial derivatives by the time and the coordinate $\mathrm{z}$.

In the next order of the perturbation theory in the small parameter $\left(\nu \tau_{0}\right)^{-1}<<1$ (here $\tau_{0}=H / v_{T}$ - characteristic time) we find connections:

$$
M_{4}=-\frac{5 v_{T}}{2 \nu} M_{3 z}, \quad M_{5}=\frac{v_{T}}{\nu} M_{2 z}, \quad M_{6}=0,
$$

substituting which into the system of first three equations (7) one arrives at the linearized system of Navier - Stokes equations:

$$
\begin{aligned}
& M_{1 t}+\frac{v_{T}}{2} M_{2 z}-\frac{v_{T}}{2 H} M_{2}=0, \\
& M_{2 t}+v_{T}\left(M_{1}+M_{3}\right)_{z}-\frac{v_{T}}{H} M_{3}-\frac{2}{3} \frac{v_{T}^{2}}{\nu} M_{2 z z}=0, \\
& M_{3 t}+\frac{v_{T}}{3} M_{2 z}-\frac{5}{6} \frac{v_{T}^{2}}{\nu} M_{3 z z}=0 .
\end{aligned}
$$


In the higher orders of the theory from the system (17) $\mathrm{s}$ the linearized Barnett's equations follow. For example, in the third order of the small parameter $\left(\nu \tau_{0}\right)^{-1}<<1$ we have:

$$
\begin{aligned}
& M_{1 t}+\frac{v_{T}}{2} M_{2 z}-\frac{v_{T}}{2 H} M_{2}=0, \\
& M_{2 t}+v_{T}\left(M_{1}+M_{3}\right)_{z}-\frac{v_{T}}{H} M_{3}-\frac{2}{3} \frac{v_{T}^{2}}{\nu} M_{2 z z}-\frac{v_{T}^{3}}{\nu^{2}}\left(M_{1 z}+\frac{1}{H} M_{1}-\frac{1}{H} M_{3}\right)_{z z}=0, \\
& M_{3 t}+\frac{v_{T}}{3} M_{2 z}-\frac{5}{6} \frac{v_{T}^{2}}{\nu} M_{3 z z}-\frac{v_{T}^{3}}{\nu^{2}}\left(M_{2 z}-\frac{1}{H} M_{2}\right)_{z z}=0 .
\end{aligned}
$$

\section{A free molecular flow limit.}

Next we would study the opposite limiting case - so-called Knudsen regime $\left(\nu<<1 / \tau_{0}\right)$. For simplicity we restrict ourselves now by a consideration of stationary solutions of the systems (7).

At $\nu=0$ ( collisionless gas) the general solution of the system (17) is expressed in elementary functions

$$
\begin{aligned}
M_{1} & =C_{1}+C_{3} \frac{z}{H}-3 C_{5} \exp \left(-\frac{z}{2 H}\right), & M_{2} & =C_{2} \exp \left(\frac{z}{H}\right), \\
M_{3} & =C_{3}-\frac{1}{3} C_{5} \exp (-z 2 H), & M_{4} & =\left(C_{4}-C_{2} \frac{z}{H}\right) \exp \left(\frac{z}{H}\right), \\
M_{5} & =C_{5} \exp \left(-\frac{z}{2 H}\right), & M_{6} & =\frac{1}{9}\left(2 C_{4}-7 C_{2}-5 C_{6}\right) \exp \left(\frac{z}{H}\right) .
\end{aligned}
$$

Generally, when $\nu \neq 0$, the solutions for $M_{2}$ and $M_{4}$ remain the same, while for $M_{5}$ it is possible to derive the linear non-uniform equation with variable coefficients

$$
M_{5 z z}+M_{5 z} \frac{1}{2 H}-M_{5} \frac{5}{3} \frac{\nu_{0}^{2}}{v_{T}^{2}} \exp \left(-\frac{2 z}{H}\right)=-\frac{7 \nu_{0}}{3 v_{T} H} C_{2} .
$$

Leaving borders of the system motionless, let's assume that average speed of the gas $M_{2}=0$. Then, if $C_{2}=0$, we go to the linear homogeneous equation for the moment $M_{5}$. In dimensionless variables $z \rightarrow z / H$ it is:

$$
M_{5 z z}+\frac{1}{2} M_{5 z}-K e^{-2 z} M_{5}=0
$$


where

$$
K=\frac{5}{3}\left(\frac{\nu_{0} H}{v_{T}}\right)^{2}=\frac{5}{3}(K n)^{-2}
$$

Let's change the coordinate variable as $\exp (-2 z)=t$, then the equation (9) becomes:

$$
t M_{5 t t}+\frac{3}{4} M_{5 t}-k M_{5}=0
$$

where $k=\frac{5}{12} \frac{\nu_{0}^{2} H^{2}}{v_{T}^{2}}$.

The equation (10) represents the linear differential equation ( degenerate hypergeometric one) of the second order with variable coefficients. The solution may be found as the generalized power series

$$
M_{5}(t)=\sum_{n=0}^{\infty} a_{n}^{(5)} t^{n}+t^{\frac{1}{4}} \sum_{n=0}^{\infty} b_{n}^{(5)} t^{n}
$$

Returning to the former dimensionless variable $\bar{z}=\frac{z}{H}$ one arrives at:

$$
M_{5}(\bar{z})=\sum_{n=0}^{\infty} a_{n}^{(5)} \exp (-2 n \bar{z})+\exp \left(-\frac{\bar{z}}{2}\right) \sum_{n=0}^{\infty} b_{n}^{(5)} \exp (-2 n \bar{z}) \text {. }
$$

For the coefficients $a_{n}^{(5)}$ and $b_{n}^{(5)}$ we have recurrent formulas:

$$
a_{n+1}^{(5)}=\frac{4 k}{(n+1)(4 n+3)} a_{n}^{(5)}, \quad b_{n+1}^{(5)}=\frac{4 k}{(n+1)(4 n+5)} b_{n}^{(5)} .
$$

Substituting $M_{5}$ into the equations for other moments yields:

$$
\begin{aligned}
M_{2}(\bar{z}) & =0 \\
M_{4}(\bar{z}) & =M_{40} \exp (\bar{z}) \\
M_{3}(\bar{z}) & =M_{30}-\frac{2}{5} \frac{\nu_{0} H}{v_{T}} \bar{z} M_{40}+\frac{1}{5} a_{0}^{(5)} \bar{z}+\frac{1}{15} a_{0}^{(5)}+\sum_{n=1}^{\infty} a_{n}^{(3)} \exp (-2 n \bar{z})- \\
& -\frac{1}{3} b_{0}^{(5)} \exp \left(-\frac{\bar{z}}{2}\right)+\exp \left(-\frac{\bar{z}}{2}\right) \sum_{n=1}^{\infty} b_{n}^{(3)} \exp (-2 n \bar{z})
\end{aligned}
$$

where

$$
a_{n}^{(3)}=\frac{1}{30} \frac{2 n-3}{n} a_{n}^{(5)}, \quad b_{n}^{3}=\frac{1}{15} \frac{4 n-5}{4 n+1} b_{n}^{(5)} .
$$


Next the expressions for $M_{6}$ and $M_{1}$ :

$$
\begin{aligned}
M_{6}(\bar{z}) & =M_{60} \exp (\bar{z})+\frac{5}{9} \frac{\nu_{0} H}{v_{T}} \exp (\bar{z}) \sum_{n=0}^{\infty} b_{n}^{(6)} \exp (-\bar{z}(4 n+5) / 2)+ \\
& +\frac{5}{9} \frac{\nu_{0} H}{v_{T}} \exp (\bar{z}) \sum_{n=0}^{\infty} a_{n}^{(6)} \exp (-2 \bar{z}(n+1)) \\
M_{1}(\bar{z}) & =M_{10}+\frac{1}{5} \frac{\nu_{0} H}{v_{T}} M_{40}\left(2 \bar{z}-\bar{z}^{2}\right)+\left(\frac{3}{5}+\frac{1}{10} \bar{z}^{2}-\frac{4}{5} \bar{z}\right) a_{0}+ \\
& +3 b_{0} \exp \left(-\frac{\bar{z}}{2}\right)+M_{30}(\bar{z}-1)+\sum_{n=1}^{\infty} a_{n}^{(1)} \exp (-2 n \bar{z})+ \\
& +\exp \left(-\frac{\bar{z}}{2}\right) \sum_{n=1}^{\infty} b_{n}^{(1)} \exp (-2 n \bar{z}) .
\end{aligned}
$$

The coefficients are:

$$
\begin{aligned}
a_{n}^{(1)}=\frac{1}{20} \frac{(2 n+1)(6 n+1)}{n^{2}} a_{n}^{(5)}, & b_{n}^{(1)}=\frac{1}{5} \frac{(12 n+5)(4 n+3)}{(4 n+1)^{2}} b_{n}^{(5)}, \\
a_{n}^{(6)}=\frac{a_{n}^{(5)}}{2(n+1)}, & b_{n}^{(6)}=\frac{2 b_{n}^{(5)}}{4 n+5} .
\end{aligned}
$$

From the recurrent formulas for $a_{n}^{(5)}$ and $b_{n}^{(5)}$ it is possible to derive their expressions via constants $a_{0}^{(5)}$ and $b_{0}^{(5)}$ :

$$
\begin{aligned}
& a_{n}^{(5)}=a_{0}^{(5)} \frac{(4 k)^{n}}{n ! \cdot \underbrace{3 \cdot 7 \cdot 11 \cdot \ldots(4 n+3)}_{n}} \\
& b_{n}^{(5)}=b_{0}^{(5)} \frac{(4 k)^{n}}{n ! \cdot \underbrace{5 \cdot 9 \cdot 13 \cdot \ldots(4 n+5)}_{n \text { factors }}}
\end{aligned}
$$

The series determining the solution (12) and (13), converge at any $z \neq 0$. Behavior of the moments $M_{n}$ as functions of $\bar{z}$ are shown in figures Fig 1 . Some discussion of this stationary case that could be considered as the ground state of Knudsen atmosphere theory is published at [32], where a verification of the theoretical results are made via molecular dynamics simulations. The deviations of exponential behavior of such atmosphere were discussed also in 33, 


\section{Construction of solutions of the momenta system by WKB method.}

In this section we apply the method WKB to the system (17). We shall assume, that on the bottom boundary at $z=0$ a wave with characteristic frequency $\omega_{0}$ is generated. Next we choose the frequency $\omega_{0}$ to be large enough, to put characteristic parameter $\xi=\frac{3 \omega_{0} H}{v_{T}} \gg 1$. We shall search for the solution in the form:

$$
M_{n}=\psi_{n} \exp \left(i \omega_{0} t\right)+c . c .
$$

where, for example, $\psi_{1}$, corresponding to the moment $M_{1}$, is given by the expansion:

$$
\psi_{1}=\sum_{k=1}^{6} \sum_{m=1}^{\infty} \frac{1}{(i \xi)^{m}} A_{m}^{(k)} \exp \left(i \xi \varphi_{k}(z)\right),
$$

here $\varphi_{k}(z)$ - the phase functions corresponding to different roots of dispersion relation. For other moments $M_{n}, \quad n=2, \ldots, 6$ corresponding functions $\psi_{n}$ are given by similar to (15) expansion. The appropriate coefficients of the series we shall designate by corresponding $B_{m}^{(k)} C_{m}^{(k)} D_{m}^{(k)} E_{m}^{(k)} F_{m}^{(k)}$. Substituting the series (15) at the system (17) one arrives at algebraic equations for the coefficients of (15) in each order. The condition of solutions existence results in the mentioned dispersion relation:

$$
\begin{aligned}
& 6 \eta^{3}+\left(5 u^{2}+20 i u-21\right) \eta^{2}+\left(5 i u^{3}-24 u^{2}-33 i u+10\right) \eta- \\
& -3 i u^{3}+9 u^{2}+9 i u-3=0
\end{aligned}
$$

Here for convenience the following designations are entered:

$$
\left(\frac{\partial \varphi_{k}}{\partial z}\right)^{2}=\frac{2}{9} \eta_{k}, \quad u=\frac{\nu_{0}}{\omega_{0}} \exp (-\bar{z}) .
$$

For the coefficients $A_{1}^{(k)} B_{1}^{(k)} \ldots$ the algebraic relations are obtained:

$$
\begin{aligned}
& B_{1}^{(k)}=\mp \sqrt{\frac{2}{\eta_{k}}} A_{1}^{(k)}, \quad C_{1}^{(k)}=\frac{1}{3} \frac{4 i u-1+\eta_{k}}{2 i u+3 \eta_{k}} A_{1}^{(k)}, \quad D_{1}^{(k)}= \pm \frac{1}{2} \frac{1+5 \eta_{k}}{2 i u+3 \eta_{k}} \sqrt{\frac{2}{\eta_{k}}} A_{1}^{(k)} \\
& E_{1}^{(k)}=-\frac{1}{\eta_{k}} \frac{3 i u-5 i u \eta_{k}+5 \eta_{k}-5 \eta_{k}^{2}}{2 i u+3 \eta_{k}} A_{1}^{(k)}, \quad F_{1}^{(k)}=\mp \frac{i \sqrt{2 \eta_{k}}}{3 u} E_{1}^{(k)} .
\end{aligned}
$$


The dispersion relation (16) represents the cubic equation with variable coefficients, therefore the exact analytical solution by formula Cardano looks very bulky and inconvenient for analysis. We study the behavior of solutions at $\nu \rightarrow 0$ (free molecular regime) and $\nu \rightarrow \infty$ (a hydrodynamical regime).

At $\nu=0$ the dispersive relation becomes:

$$
6 \eta^{3}-21 \eta^{2}+10 \eta-3=0 .
$$

The roots are:

$$
\eta_{1}=3, \quad \eta_{2} \approx 0.25+0.32 i, \quad \eta_{3} \approx 0.25-0.32 i
$$

Specifying roots (16) by the theory of perturbations up to $u^{3}$ for the three solutions branches it is obtained:

$$
\begin{aligned}
& \eta_{1}=3-0.56 u^{2}-i\left(1.96 u-0.17 u^{3}\right) \ldots, \\
& \eta_{2}=(0.25+0.32 i)+(0.07-0.69 i) u-(0.14-0.52 i) u^{2}+(0.19-0.08 i) u^{3} \ldots, \\
& \eta_{3}=(0.25-0.32 i)-(0.07+0.69 i) u-(0.14+0.52 i) u^{2}+(0.19+0.08 i) u^{3} \ldots
\end{aligned}
$$

Correspondingly for $\varphi_{z k}=\frac{\sqrt{2}}{3} \sqrt{\eta_{k}}$ we have:

$$
\begin{aligned}
\varphi_{z 1} & =1.15-0.47 u^{2}-i\left(0.37 u-0.17 u^{3}\right) \ldots, \\
\varphi_{z 2} & =(0.38+0.19 i)-(0.12+0.34 i) u+(0.12+0.02 i) u^{2}+(0.06+0.07 i) u^{3} \ldots, \\
\varphi_{z 3} & =(0.38-0.19 i)+(0.12-0.34 i) u+(0.12-0.02 i) u^{2}-(0.06-0.07 i) u^{3} \ldots
\end{aligned}
$$

Similarly in a limit $\nu \rightarrow \infty$ (a hydrodynamical limit) for solutions of the equation (16) $\eta_{k}$ it is derived:

$$
\begin{aligned}
& \eta_{1}=0.6-1.87 u^{-2}-i\left(0.72 u^{-1}-4.27 u^{-3}\right) \\
& \eta_{2}=-i\left(u+0.80 u^{-1}\right)+1.40+0.18 u^{-2} \\
& \eta_{3}=-0.83 u^{2}+1.50-i\left(2.33 u-1.52 u^{-1}\right) .
\end{aligned}
$$

The first root relates to the acoustic branch. Accordingly, for the $\varphi_{z k}$ we have:

$$
\begin{aligned}
& \varphi_{z 1} \approx 0.52-0.71 u^{-2}-i\left(0.31 u^{-1}-1.41 u^{-3}\right) \ldots \\
& \varphi_{z 2} \approx \sqrt{u}(1-i)\left(0.47+0.30 u^{-2}\right)+\sqrt{u}(1+i)\left(0.33 u^{-1}+0.07 u^{-3}\right) \ldots, \\
& \varphi_{z 3} \approx-0.85+0.62 u^{-2}+i\left(0.61 u+0.05 u^{-2}\right) \ldots
\end{aligned}
$$


The solution of the equation (16) at any $u$ is evaluated numerically. Behavior of real and imaginary parts $\eta_{n}$ and $\varphi_{z k}$ as functions of $u$, and their behavior on a complex plane are presented at the figures 1 - 4 .

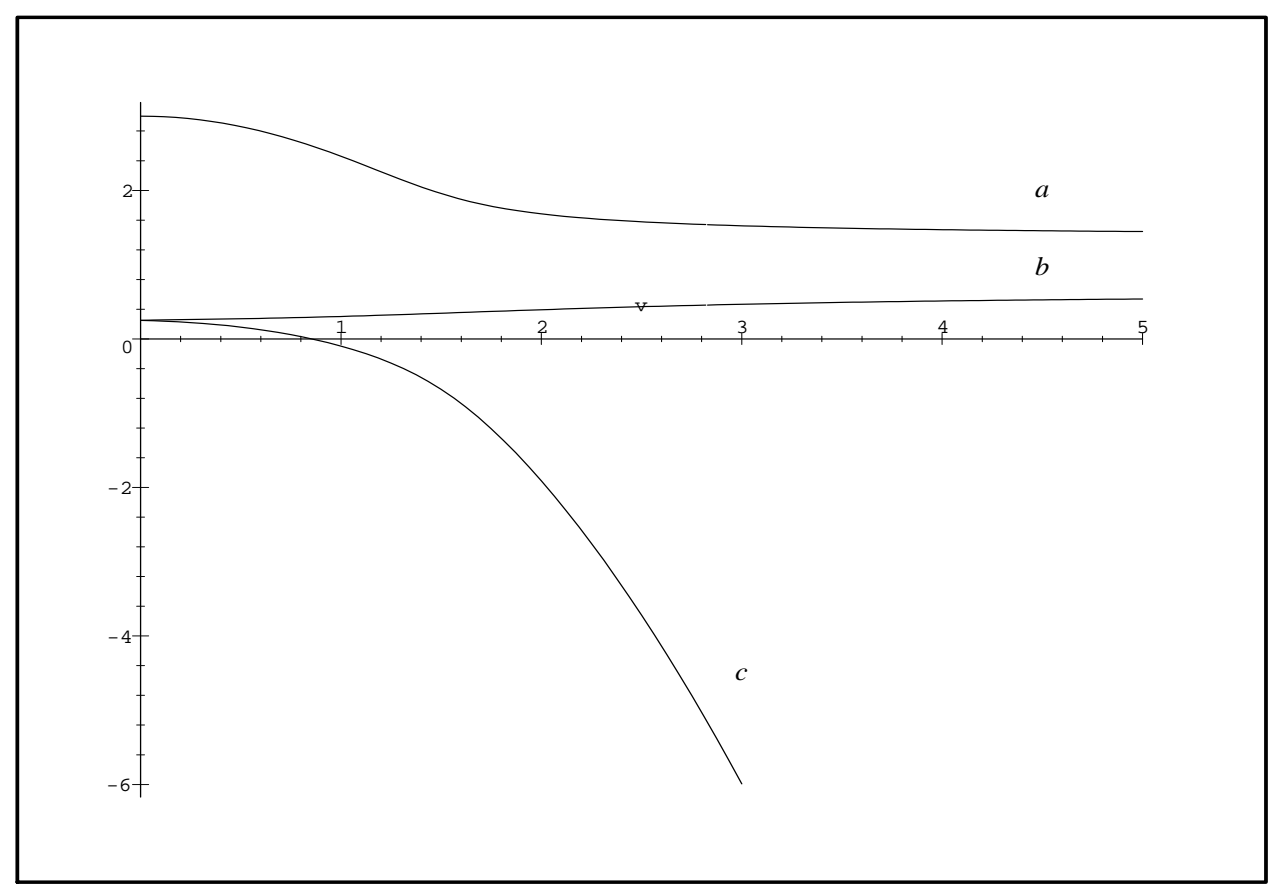

Fig. 1. Behavior of the real part of roots of a dispersion relation:

$$
\mathrm{a}-\eta_{1}, \mathrm{~b}-\eta_{2}, \mathrm{c}-\eta_{3}
$$




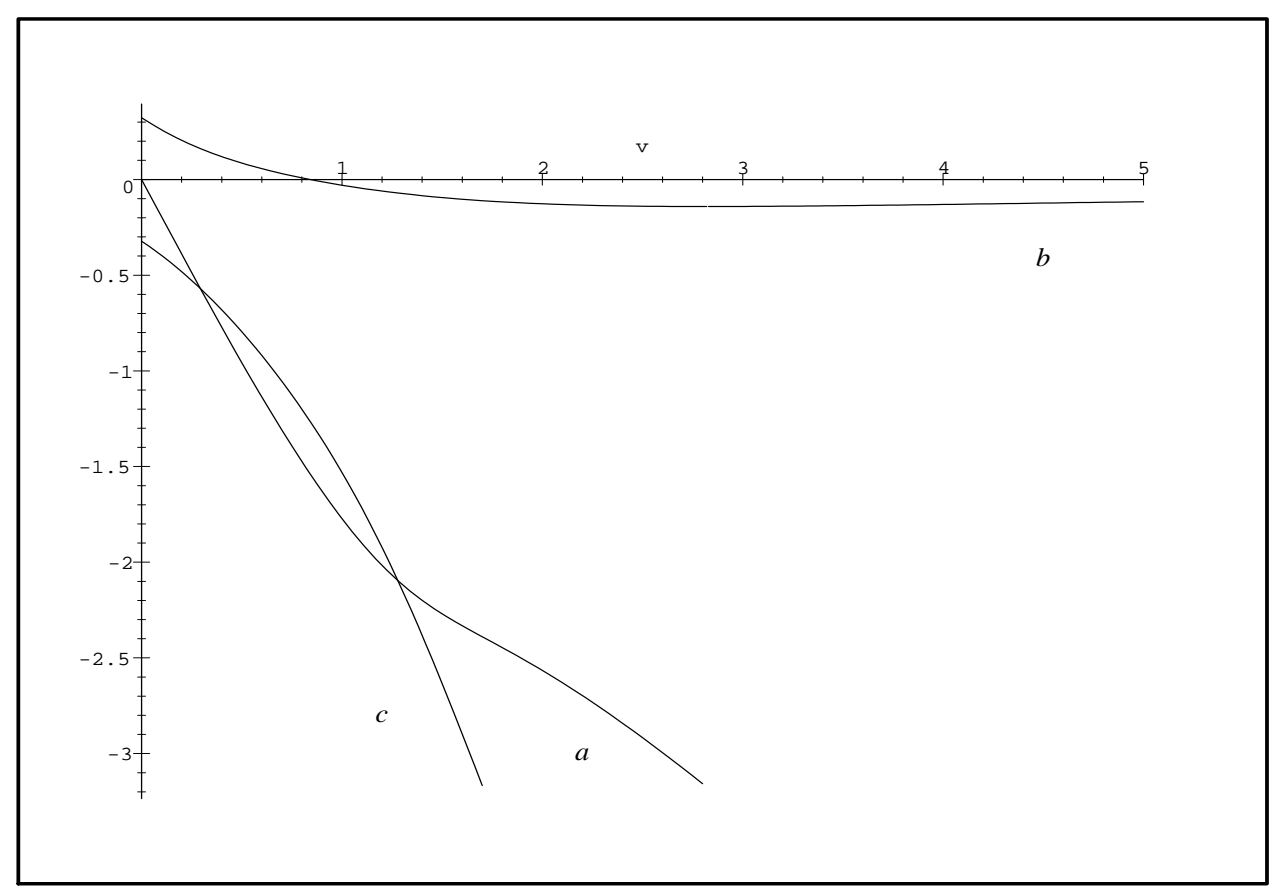

Fig. 2. Behavior of the imaginary part of the roots of the dispersive relation:

$$
\mathrm{a}-\eta_{1}, \mathrm{~b}-\eta_{2}, \mathrm{c}-\eta_{3}
$$




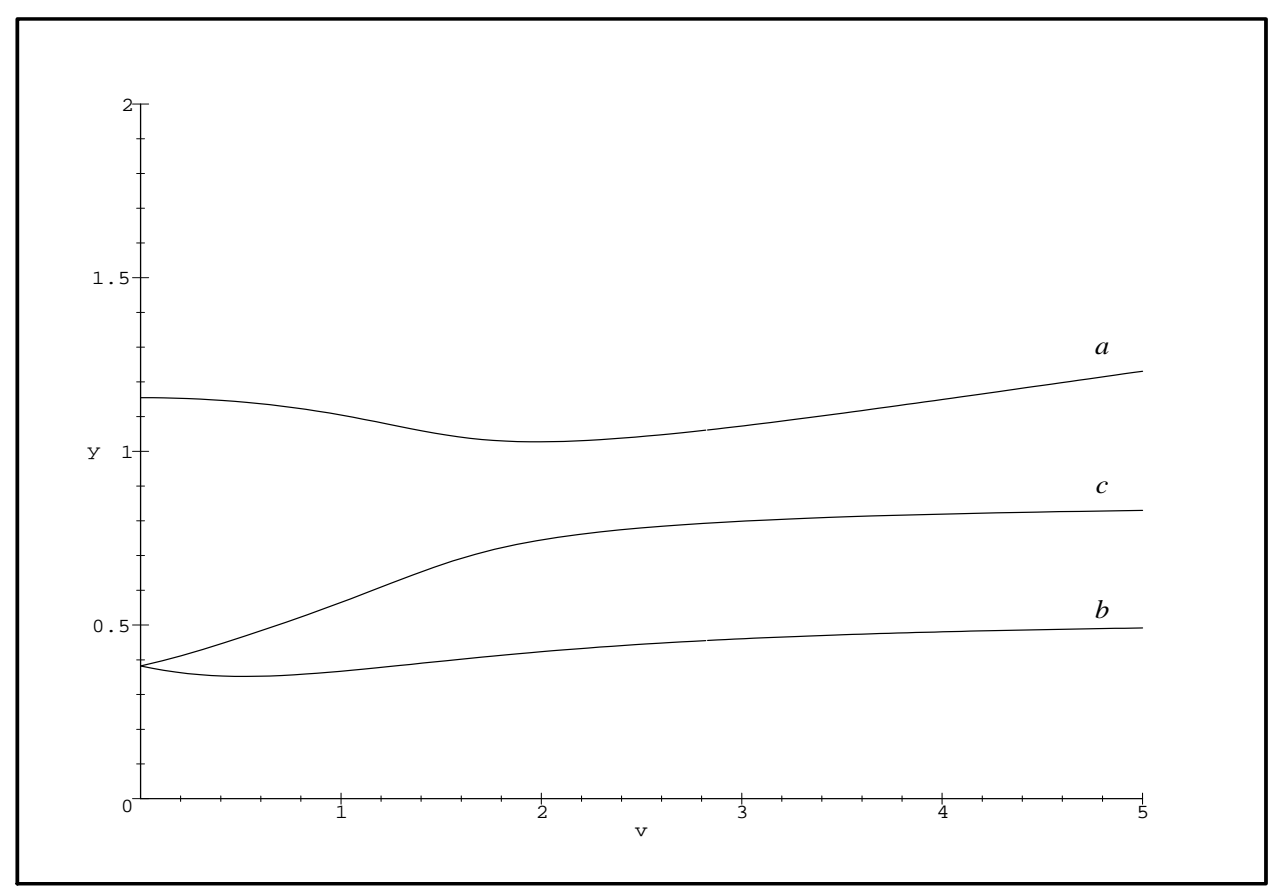

Fig. 3. Behavior of the real part of the phase functions $\phi_{n z}: \mathrm{a}-\phi_{1 z}, \mathrm{~b}-\phi_{2 z}$, $c-\phi_{3 z}$ 


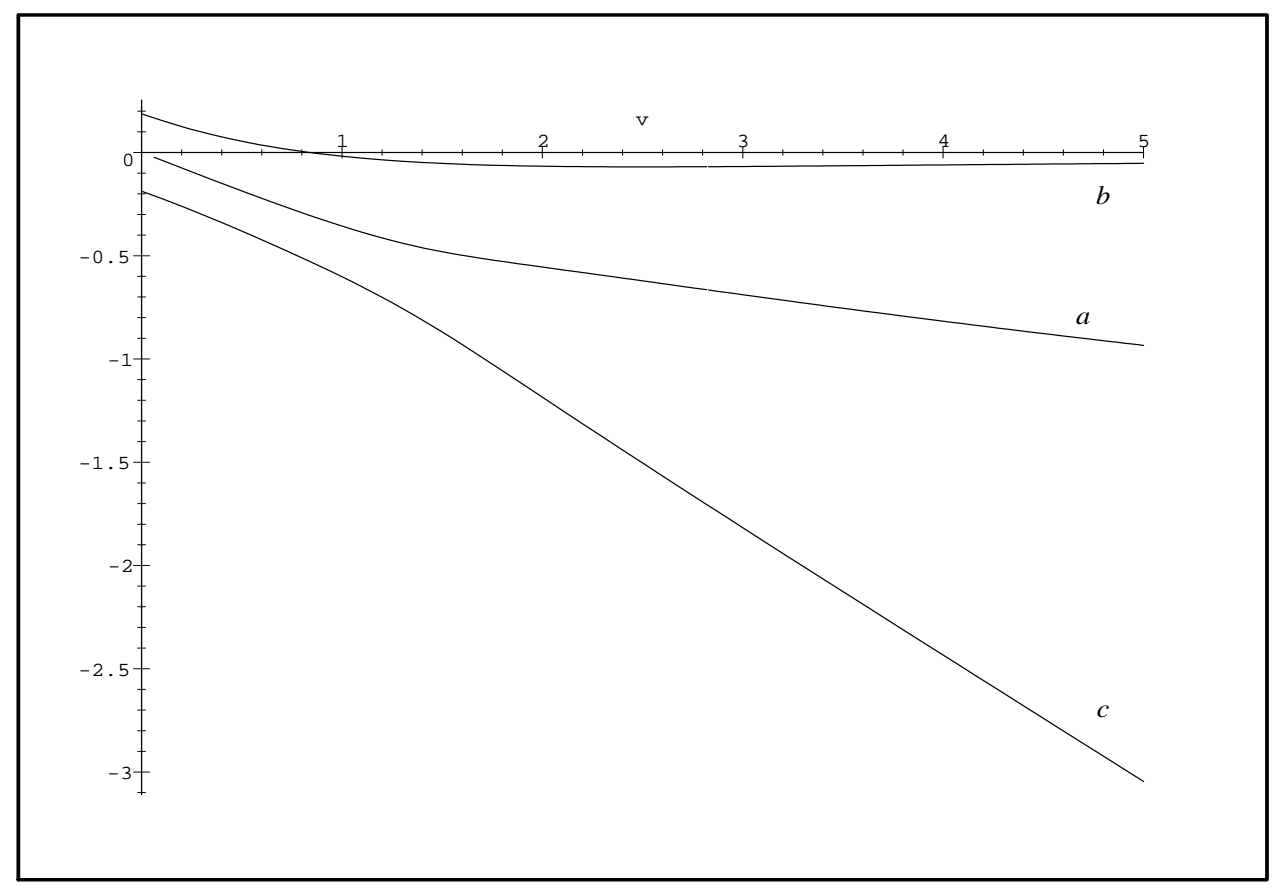

Fig. 4. Behavior of the imaginary part of the phase functions $\phi_{n z}$ :

$$
\mathrm{a}-\phi_{1 z}, \mathrm{~b}-\phi_{2 z}, \mathrm{c}-\phi_{3 z}
$$

As an illustration let us consider a problem of generation and propagation of a gas disturbance, by a plane oscillating with a given frequency $\omega_{0}$. Let's assume, that at height $z=0$ all moments $M_{k}=0$, except for $M_{2}=U_{0}$. In evaluations we we choose the dimensionless frequency equal to $u(0)=0.1$, that fits heights $z 300$ in the Earth atmosphere. Behavior of the solutions for $M_{k}$ constructed by the VKB technique are shown on fig. 7 . 


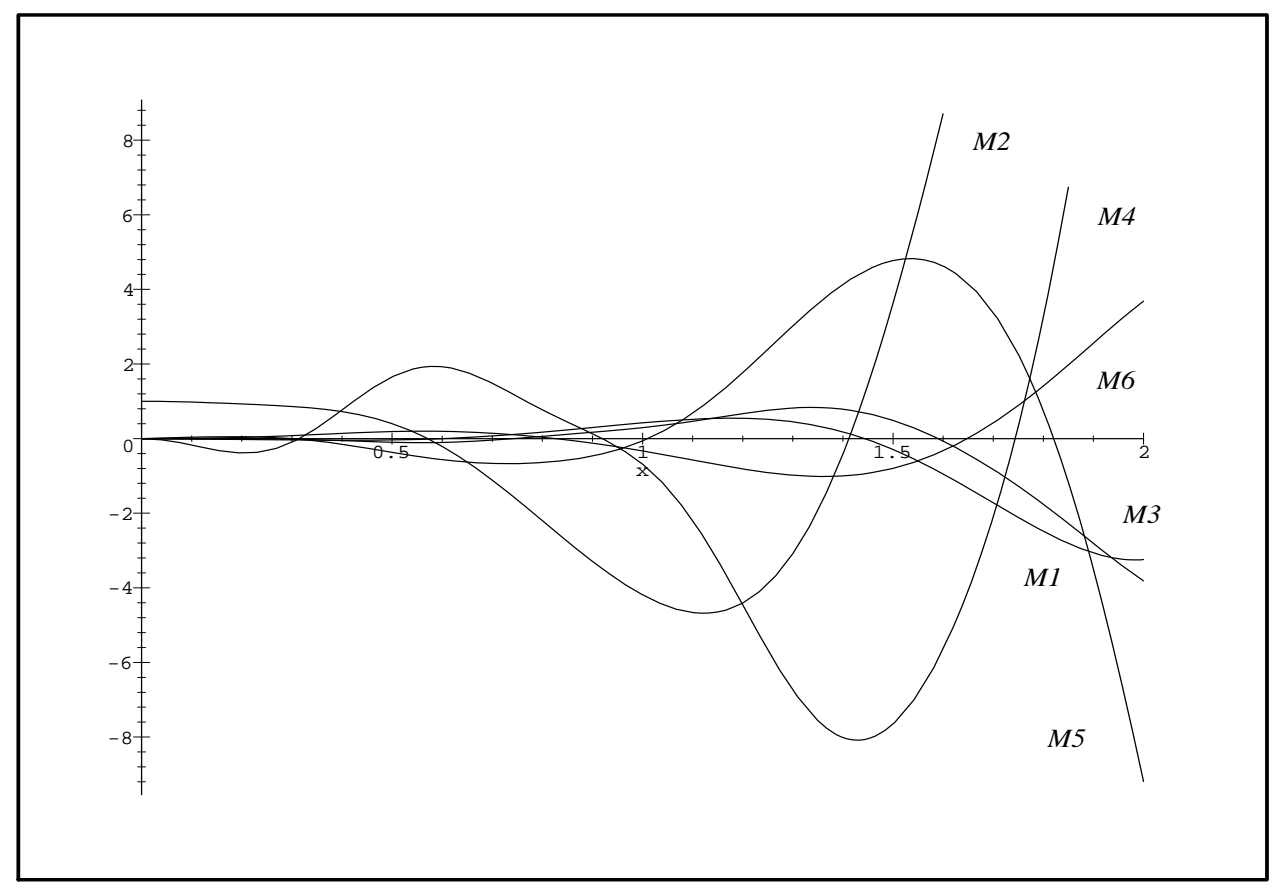

Fig. 5. Dependence of the moments $M_{k}$ on height, $u(0)=0.1$ :

\section{Conclusion}

In this paper we propose a one-dimensional theory of linear disturbances in a gas, stratified in gravity field, hence propagating through regions with crucially different Kn numbers. The regime of the propagation dramatically changes from a typically hydrodynamic to the free-molecular one. We also studied three-dimensional case, to be published elsewhere. The theory is based on BGK or Gross-Jakson kinetic equation, which solution is built by means of locally equilibrium distribution function with different local parameters for molecules moving "up" and "down". Equations for six moments yields in the closed fluid mechanics system. For the interesting generalizations of the foundation of such theory see the recent review of Alexeev [34]. 


\section{Acknoledgement}

We would like to thank M. Solovchuk for important technical help.

\section{References}

[1] Wang Chang C.S., Uhlenbeck G.E. On the propagation of sound in monoatomic gases. Eng.Res.Ins., Univ. of Michigan.Project M 999. Ann.Arbor., Michigan. (1952).

[2] Foch D., Ford Jr.G.M. The description of sound in monoatomic ases. In "Stadies in Statistical Mechanics" (ed, J. de Boer and G.E. Uhlenbeck), N.Holland,5. (1970). P.103-231.

[3] Meyer E., Sessler G. Schalaus breitung in gasen bei hohoen frequenzen und sehr niedrigen drucken. Z.Physik. 149. (1957). P.15-39.

[4] Greenspan M. Propagation of sound in five monatomic gases. J.Acoust.Soc.Am.., 28. No 4. (1965). P.644-648.

[5] Backner J.K., Ferziger J.H. Linearized initial value problem for a gas. Phys.Fluids.9. № 12. (1966). P.2309-2314.

[6] Backner J.K., Ferziger J.H. Linearized boundary value problem for a gas and sound propagation. Phys.Fluids.9. № 12. (1966). P.2315-2322.

[7] Sirovich L., Thurber J.K. Sound propagation according to kinetic models. Inst.Math¿Soc.Rept. AFOSP-1380. MF-17. New York UNiv. (1961).

[8] Sirovich L., Thurber J.K. Sound propagation according to the kinetic theory of gases. Adv.Appl.Mech.,Supp.2. 1. (1963). P.152-180.

[9] Sirovich L., Thurber J.K. Propagation of forced sound waves in rarefied gas dynamics. Acoust.Soc.Am.37. № 2. (1965). P.329-339.

[10] Sirovich L., Thurber J.K. Plane wave propagation in kinetic theory. J.Math.Phys.8. No 4. (1967). P.888-895.

[11] Thomas J.R., Siewert G.E. Sound wave propagation in a rarified gas. Trans.Theory and Stat.Phys.,8.(1079). P.219-240. 
[12] Loyalka S.K., Cheng T.S. Sound wave propagation in a rarified gas. Phys.Fluids.,22. № 5. (1979). P.830-836.

[13] Cheng T.S., Loyalka S.K. Sound wave propagation in a rarified gas. II. Gross-Jackson model. Progress in Nuclear Energy. 8. (1981). P.236-267.

[14] Monchik L. Small periodic disturbances in polyatomic gases. Phys.Fluids. 7. № 6. (1964). P.882-896.

[15] Banankhah A., Loyalka S.K. Propagation of a sound wave in a rarified polyatomic gas. Phys.Fluids.30. № 1. (1987). P.56-64.

[16] Leble S.B., Vereshchagin D.A. Kinetic description of sound propagation in exponentially stratified media. Advances in Nonlinear Acoustic (ed.H.Hobaek).Singapore. World Scientific. (1993). P.219-224.

[17] Vereshchagin D.A., Leble S.B. Stratified gas and nonlinear waves passing the Knudsen layer. Proceedings of International Symposium on Nonlinear Theory and its Applications "NOLTA '93".(Hawaii,1993). 3. (1993). P.1097-1100.

[18] Vereshchagin D.A., Leble S.B. Piecewise continuous partition function and acoustics in stratified gas. Nonlinear Acoustics in Perspective, ed. R.Wei, (1996),p.142-146.

[19] X Chen, H Rao, E Spiegel Macroscopic equations for rarefied gas dynamics. Phys. Lett. A 271 (2000) 87-91.

[20] X. Chen, H. Rao, and E. A. Spiegel, "Continuum description of rarefied gas dynamics: II. The propagation of ultrasound," Phys. Rev. E 64, 046309 (2001).

[21] S. Leble Nonlinear Waves in Waveguides (Springer-Verlag, 1991),164p.

[22] Lees L. Kinetic theory description of rarefied gas flow. J.Soc.Industr. and Appl.Math.,13.№ 1. (1965) P.278-311.

[23] Vereshchagin D.A., Shchekin A.K., Leble S.B. Boundary regime propagation in a stratified gas with arbitrary Knudsen number. Zhurnal Prikl. Mech. and Tehn. Fiz., No5. P.70-79. (in Russian). 
[24] Shchekin A.K., Leble S.B., Vereshchagin D.A. Introduction in physical kinetic of rarefied gas. Kaliningrad. (1990) 80.p. (in Russian).

[25] Liu Chung Yen., Lees L. in"Rarefied gas dynamics" (ed.by L.Talbot). Academic Press. (1961). P.391-428.

[26] Yang H.T., Lees L. Journ.Math.Phys.,35.(1956) P.195-235.

[27] Shidlovskij I.P. The introduction in rarified gas dynamics. Moscow, Nauka. (1965) 220.p. (in Russian). Ing. Journ. 3. No 3. (1963) (in Russian).

[28] Mott-Smith H.M. The solution of the Boltzmann equation for a shock wave. Phys.Rev.,82. (1951).P.885-892.

[29] Nambu K., Watanabe Y. Analysis of the internal structure of shock waves by means of the exact direct-simulation method. Rep.Inst.High Speed.Mech., 48. № 366.(1984).P.1-75.

[30] Sampson R.E., Springer G.S. Condensation on and evaporation from droplets by a moment method. J.Fluids.Mech. 36. part.3. (1969).P.577584 .

[31] Ivchenko I. Evaporation (condensation) theory of spherical particles with all Knudsen number. J.Coll and Interf.Sience. 120. № 1. (1987). P.1-7.

[32] Leble S.B, F.L. Roman, D.A. Vereshchagin and J.A. White. Molecular Dynamics and Momenta BGK Equations for Rarefied Gas in Gravity field. in Proceedings of 8th Joint EPS-APS International Conference Physics Computing CYFRONET-KRAKOW, Ed. P.Borcherds, M.Bubak, A.Maksymowicz (1996), p.218-221.

[33] Román F.L., White J.A.and Velasco S.: On a paradox concerning the temperature distribution of an ideal gas in a gravitational field. Eur. J. Phys. 16 (1995) 83-90

[34] B.V. Alexeev Physical principles of generalized Boltzmann kinetic theory. Uspechi Fiz. Nauk 170 2000, p649-679. 
<smiles>C1C2C3CC4C1C1C2C3C41</smiles> 


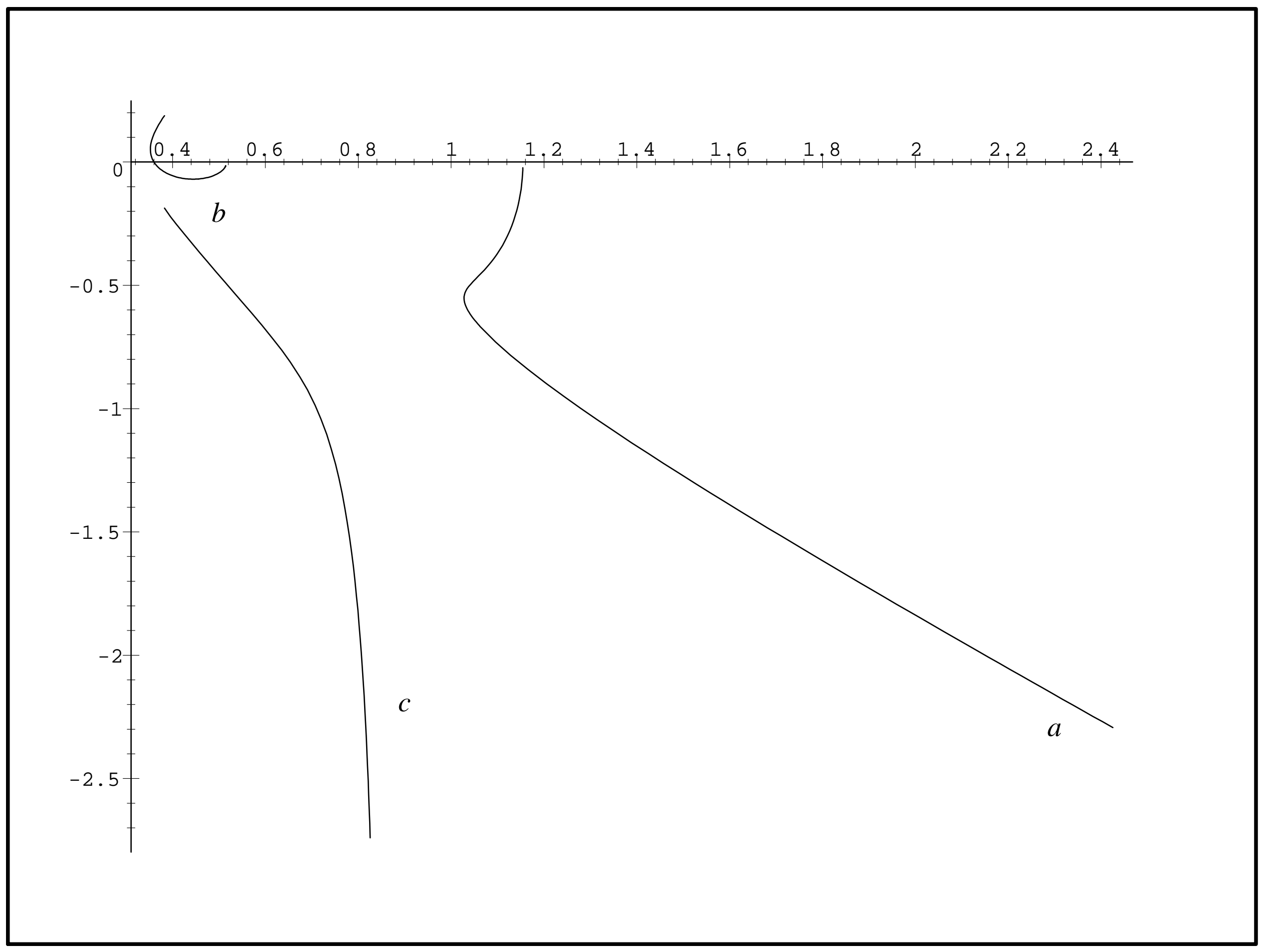

